\title{
UAV Formation Control: Theory and Application ${ }^{\star}$
}

\author{
Brian D.O. Anderson ${ }^{1}$, Barış Fidan ${ }^{1}$, Changbin $\mathrm{Yu}^{1}$, and Dirk van der \\ Walle $^{2}$ \\ 1 Research School of Information Sciences and Engineering, The Australian \\ National University and National ICT Australia, Canberra, Australia \\ \{Brian. Anderson, Baris.Fidan, Brad.Yu\}@anu. edu. au \\ 2 Delft Center for Systems and Control, Delft University of Technology, Delft, the \\ Netherlands D.vanderWalle@student.tudelft.nl
}

Summary. Unmanned airborne vehicles (UAVs) are finding use in military operations and starting to find use in civilian operations. UAVs often fly in formation, meaning that the distances between individual pairs of UAVs stay fixed, and the formation of UAVs in a sense moves as a rigid entity. In order to maintain the shape of a formation, it is enough to maintain the distance between a certain number of the agent pairs; this will result in the distance between all pairs being constant. We describe how to characterize the choice of agent pairs to secure this shape-preserving property for a planar formation, and we describe decentralized control laws which will stably restore the shape of a formation when the distances between nominated agent pairs become unequal to their prescribed values. A mixture of graph theory, nonlinear systems theory and linear algebra is relevant. We also consider a particular practical problem of flying a group of three UAVs in an equilateral triangle, with the centre of mass following a nominated trajectory reflecting constraints on turning radius, and with a requirement that the speeds of the UAVs are constant, and nearly (but not necessarily exactly) equal.

Key words: Formation control, surveillance, UAV, rigid formation, persistent formation.

\section{Introduction}

Today, technology allows us to mimic the behavior of insects, animals, birds, etc. $[1,2]$ using robots, unmanned airborne vehicles (or indeed regular aircraft,

\footnotetext{
* This work is supported by National ICT Australia, which is funded by the Australian Government's Department of Communications, Information Technology and the Arts and the Australian Research Council through the Backing Australia's Ability Initiative.
} 
underwater autonomous vehicles, and the like). The range of applications is steadily growing, and it includes military and civilian applications, very often involving surveillance or exploration of some region.

Why use a formation? When the agents in a formation, unmanned airborne vehicles (UAVs) for example, are engaged in surveillance or exploration activity, they are typically able to synthesize an antenna of dimension far larger than an individual agent. The benefit is the improved sensitivity. If source localization is of concern, not only is the improved sensitivity beneficial, but additionally, some localization tasks inherently require multiple sensors with known relative positions. A different reason is that multiple sensors may have different functionalities, and the aggregate functionality may give a new functionality for the formation of sensors. Again, many sensors, such as UAVs or sensors in a sensor network, are weight-constrained, and small mobile sensors can be cheaper to deploy.

Sometimes several of these factors can be simultaneously operative. We consider later in this chapter by way of a case study an application involving a cooperative UAV surveillance task. The agents determine angle information associated with the object at an unknown position. There is sensor noise and a limited cone of visibility. So more agents need to be used in the formation than might at first be imagined.

Control of a formation requires the mixing of several tasks. One is the whole formation task of moving from point A to point B (or moving the centre of mass of the formation, and adopting a certain orientation). Another is to maintain the relative positions of the agents during formation motion, so that the shape is preserved. A third might be to avoid obstacles. A fourth might be to split the formation, etc. Generally speaking, in nature and a number of man-made systems, there is no single master agent which oversights or controls every other agent. Some kind of decentralized handling of the control task is needed, and therein lies much of the scientific challenge associated with artificial formations.

Indeed, the highest level problem seen from the viewpoint of a control engineer is probably how to define practical architectures for the formation, or the dependencies and signal flows associated with communications, sensing and control. A key requirement is that any architecture be scalable. The number of communication links required for a single agent should not grow linearly with the number of agents in the formation for example. This scalability requirement is indeed a driver of the decentralized approach. It is clear that nature respects this too. No one bird in a formation of birds would be expected to watch every other bird, and determine where it will fly using its perception of where every other bird in the formation is flying. In this chapter, we mainly focus on the question of what sort of sensing and control architectures are needed to maintain the shape of a formation, while the formation moves as a cohesive whole. We shall present some details on types of control law that can ensure such motion occurs. 
Furthermore, we will impose yet one more level of limitation. Clearly, if a group of agents is maintaining formation shape, each must know the desired value of certain geometric variables and must sense the actual values of those geometric variables, in order to generate any corrective control. Many variables can be sensed, generally involving some combination of distances and angles, and it is possible that one agent may sense more than one class of measurement. See, for example, [3]. For the most part, we shall contemplate two dimensional formations and require that a typical agent in two dimensions use at least two distance measurements to neighboring agents to maintain its position (and often also sensing the relative position of these neighboring agents) assuming it has no degrees of freedom through being a leader or coleader of the formation ${ }^{3}$.

The chapter is organized as follows. In Section 2, we present and elaborate two different control structures that can be used in maintaining shapes of autonomous formations, the symmetric and asymmetric control structures, and characteristics of autonomous formations controlled by such control structures. Two particular graph theoretical notions are introduced and used in analysis in this section: graph (formation) rigidity and persistence. Using such ideas, in Section 3, we summarize some recent results on decentralized control design to ensure preservation of the shape of a formation, particularly focusing on autonomous formations with asymmetric control structure. Sections 4-6 are dedicated to a particular autonomous formation control application: cooperative surveillance with a team of three UAVs. Section 4 introduces the particular cooperative surveillance task considered and presents the specifications of the corresponding formation control problem. A distributed control scheme for this problem is proposed with details in Section 5. The proposed control scheme is analyzed for a number of scenarios via simulations in Section 6. As will become evident in these later sections, we draw on various aspects of the rather idealized studies of Section 3 in order to cope with the practical constraints imposed in the application. The chapter finishes with a brief summary and concluding remarks in Section 7 .

\section{Autonomous Formations and Shape Maintenance}

When distance control between pairs of agents is used, there are two approaches to controlling each distance, symmetric and asymmetric. Formations with symmetric control structure can be represented with undirected graphs, while directed graphs are used to represent those with asymmetric control structure.

\footnotetext{
${ }^{3}$ When an agent is required to maintain distances from two other agents, it will generally need relative position information, or equivalently distances to the two agents and the angle between them. The angle information may be derivable by running a Kalman filter using distance measurements, or using an additional distance measurement, viz. that between its two neighbors.
} 
When a symmetric control structure is used, to keep the distance between each pair $\left(A_{1}, A_{2}\right)$ of neighbor agents, there is a joint effort of both agents $A_{1}$ and $A_{2}$ to simultaneously and actively maintain their relative positions. The underlying graph of the formation will have an undirected edge between vertices 1 and 2 (which represent agents $A_{1}$ and $A_{2}$ ). Evidently, if enough agent pairs explicitly maintain distances, all inter-agent distances and hence the formation shape will be maintained, i.e. the formation will be rigid. Just what constitutes 'enough' will be explained in more detail subsequently.

In contrast, when an asymmetric control structure is used, only one of the agents in each neighbor agent pair, e.g., $A_{1}$ in the agent pair $\left(A_{1}, A_{2}\right)$, actively maintains its position relative to its neighbor. This means for the neighbor agent pair $\left(A_{1}, A_{2}\right)$ that only $A_{1}$ has to receive the position information broadcast by $A_{2}$, or sense the position of $A_{2}$ and make decisions on its own. Therefore, in the asymmetric control structure, both the overall control complexity and the communication complexity (in terms of message sent or information sensed) for the formation are reduced by half, compared to symmetric control. (One could argue however that redundancy is lost, and this may be dangerous). From a graph theory point of view, this asymmetric control structure is modelled in the associated directed graph by a directed edge from vertex 1 to vertex 2 (representing a directed constraint link from $A_{1}$ to $A_{2}$ ).

In this section we provide some detail on the characteristics of 2-dimensional formations with symmetric and asymmetric control structures, mainly using rigid graph theory $[4,5]$. Some but not all of the ideas are valid for 3dimensional formations. However, we shall omit discussion of the 3 -dimensional case in the interest of brevity.

\subsection{Formations with Symmetric Control Structure}

To fit within the (rigid) graph theory framework, a multi-agent system model is used where agents are assumed to be points in $\Re^{2}$, and agent pairs for which the inter-agent distance is actively constrained to be constant are thought of as being joined by bars with lengths enforcing the inter-agent distance constraints $[6,7,8,9]$. Evidently, the multi-agent system can be modeled by a graph: vertices represent point-like agents and edges join vertex pairs corresponding to agent pairs with prescribed inter-agent distance constraints. (Naturally, one can contemplate other constraints than just distance, e.g. those involving angle, or angle and distance, although discussion is omitted in this chapter for the sake of simplicity.) Rigid graph theory is used to study properties of graphs which ensure that the formation being modelled by the graph will be rigid; formal definitions are available of course, but in rough terms, a rigid formation is one in which the only smooth motions are those corresponding to translation or rotation of the whole formation.

There exist two key tool sets for rigidity analysis. Linear algebra and matroid theory provide the first: given knowledge of the positions of the agents 
at any one time, one can construct a matrix, the so-called rigidity matrix [4], and the dimensions and rank of this matrix allow one to conclude that the formation is or is not rigid. The dimensions and rank are the same for almost all positions of the agents, i.e. for generic agent positions. This means that rigidity matrices formed from two formations differing from each other only in terms of the values for the constrained distances will have the same rank, except for very special sets of the agent positions. Examples of special sets include those where two agents are at the same position, three agents are collinear, four agents form a parallelogram, etc.

The second tool set is a combinatorial one, i.e. it involves a number of purely counting-type characterization conditions. The key result is Laman's Theorem [10]. This central contribution of the rigid graph theory literature implies that it is also possible in 2 dimensions to characterize rigidity of a generic formation corresponding to a given graph in purely combinatorial conditions related to the graph (discarding the agent coordinates). By generic formation, we mean one where the agent positions are generic.

A rigid formation is further called minimally rigid if the removal of any single inter-agent distance constraint causes loss of rigidity. Unsurprisingly, a graph is called minimally rigid if almost all formations to which the graph corresponds are minimally rigid. Minimal rigidity is easily characterizable in 2-dimensions both with the rigidity matrix and with Laman's Theorem. An easily checked necessary condition is that $|E|=2|V|-3$, where $|E|$ and $|V|$ are the numbers of edges and vertices of the graph. Thus in a minimally rigid graphs the number of edges and vertices have the same order.

The formation shape of a minimally rigid formation (assuming $N>0$ agents) is maintained using the minimum possible number of information links (for the given number of agents, $N$ ), which makes use of minimally rigid information architectures an optimal (i.e. most efficient) choice. However, in many cases, there are practical reasons to use non-minimally rigid graphs rather than minimally rigid ones to underpin the shape of a formation. A minimally rigid formation offers no protection against loss of a sensor, or a communication link, or a control actuator, and in practice, it will often be necessary to obtain robustness through the use of some measure of redundancy. Measures of robustness are needed to reflect ability to sustain agent and link losses (whether from a sensing, communication or control failure) [11].

\subsection{Formations with Asymmetric Control Structure}

This subsection comments on extensions of the concepts and results presented in Section 2.1 to formations with asymmetric control structure. The basic task is again maintenance of formation shape during motion, i.e. ensuring that the smooth motions of the formation are restricted to translation or rotation. This task is examined in detail in [12] and [9].

We call a formation with asymmetric control structure persistent if it is rigid (where rigidity is as explained in Section 2.1) and satisfies another condi- 
tion termed constraint consistence [12, 9]; constraint consistence is equivalent to the requirement that it is possible to maintain the nominated inter-agent distances. (Note that the rigidity property only says that if certain interagent distances are maintained, then all inter-agent distances are maintained when the formation moves smoothly. Moreover, it is possible to assign directions to the edges of a rigid graph that render it not constraint consistent.) A minimally persistent formation is one that is minimally rigid and constraint consistent. Formal definitions and properties of constraint consistence and persistence can be found in $[12,9]$, where directed graph notions are used.

\section{Control Laws for Minimally Persistent Formations}

In this section, we summarize some recent results on the construction of decentralized control laws to ensure that the shape of a formation is preserved. The key references are $[13,14,15]$. However, we must not discount other work in this area. Among the earliest key studies linking graph theory nontrivially to problems of stabilization of the associated formations were those of OlfatiSaber and Murray [16, 17]. A very recent work with close links is [18]. This reference considers rigid and persistent formation control.

Unsurprisingly, it is generally possible to separate the navigation task for the formation (getting from A to B) from the shape stabilization task, and in this section we will focus solely on the latter task. The first of the OlfatiSaber and Murray papers provides laws based on potential functions applying to undirected graphs, and leaves open the directed case. The second of the cited papers of Olfati-Saber and Murray deals extensively with formations for which the underlying (directed) graph was acyclic. It turns out that formation stabilization in this acyclic case is more straightforward: decentralization of the control laws is easy because there is one-way-only or triangular coupling between the agents. References $[13,14,15]$ are concerned with formations where the underlying graph includes one or more cycles.

More complicated agent models than the point models used here are dealt with in some literature. See e.g. [19, 20]. We note also other problems of holding a formation of a specialized shape, e.g. equilateral polygon [21]. If all agents are equipped with a compass, stabilization is easier, [8]; that assumption will not be made here.

The key assumptions made are as follows: the formation exists in the plane, agents are point agents, with velocities controllable; agents can measure the distance to their neighbors, they know the angle between any two neighbors, and they know the desired distance to each of their neighbor(s). We restrict attention to minimally persistent formations, and we consider first the simplest formation containing a cycle-a triangular formation of three agents.

The potential for instability when a cycle is present should be reasonably clear. If agents $i, j, k$ form a cycle, $i$ tries to fix its position relative to $j, j$ tries to fix its position relative to $k$, and $k$ tries to fix its position relative to $i$, then 
there is a clear feedback mechanism around the cycle. Without analysis, one does not know if the feedback will have positive or negative effects, including destabilization.

\subsection{Control of a Triangular Formation with Three Coleaders}

This subsection summarises results of $[14,15]$. Related results can be found in [18]. Denote the three agents by 1,2 and 3 , and suppose their positions at any instant of time are denoted by $x_{i}, i=1,2,3$. Suppose the nominal distances from 1 to 2,2 to 3 and 3 to 1 are $d_{1}, d_{2}, d_{3}$, and suppose these distances satisfying the triangle inequality. Let $z_{1}, z_{2}, z_{3}$ denote the relative positions of 1 with respect to 2 , etc, i.e. $z_{1}=x_{1}-x_{2}$ with two similar equations. The formation stabilization task is to ensure that $\lim _{t \rightarrow \infty}\left\|z_{i}(t)\right\|-d_{i}=0, i=$ $1,2,3$. The question arises as to whether this behavior can reasonably be expected for all initial conditions, or only those for which ||$\left|z_{i}(t) \|-d_{i}\right|$ is small; as it turns out, it is possible to secure this behavior for almost all initial conditions. is

The control laws of $[14,15]$, while different, have a common form. The law

$$
\dot{x}_{i}=-k_{i} z_{i}
$$

In [14] and [15], the gains $k_{i}$ are respectively:

$$
\begin{array}{r}
k_{i}=\left[\left\|z_{i}\right\|-d_{i}\right] /\left\|z_{i}\right\| \\
k_{i}=\left[\left\|z_{i}\right\|^{2}-d_{i}^{2}\right]
\end{array}
$$

Clearly, movement of agent $i$ is always directly towards or directly away from its neighbor, with the direction of movement such as to reduce the error between the actual and desired distance. This is intuitively reasonable. The gain differs between the two algorithms. Actually, they are both special cases of the algorithm

$$
\dot{x}_{i}=-\phi_{i}\left(e_{i}, d_{i}\right) z_{i}
$$

where $\phi_{i}$ for fixed $d_{i}$ is a smooth first-third quadrant nonlinearity in $e_{i}=$ $\left\|z_{i}\right\|^{2}-d_{i}^{2}$ (but not defined for $e_{i}<-d_{i}^{2}$ ).

Evidently, the whole system is nonlinear in a nontrivial way, and its analysis is by no means straightforward. Despite this, much can be shown. The following properties are probably true for the general family of laws just identified; however, at the moment, the properties have just been established for one at least of the two particular laws.

Property 1. Suppose that the initial positions of all agents are such that they are not collinear. If no two of the $d_{i}$ are the same, and the second control law is used, the errors $e_{i}=\left\|z_{i}\right\|^{2}-d_{i}^{2}, i=1,2,3$ will all converge exponentially fast to zero, or equivalently the correct triangle shape is assumed exponentially fast. 
Property 2. If the initial positions result in the correct triangle shape being assumed exponentially fast, then the total translation of the triangle's centre of mass, and the total rotation of the triangle about the centre of mass over the interval $[0, \infty)$ are bounded, and all agents come to rest.

Property 3. If the initial positions of the agents are collinear, the subsequent trajectory will retain the collinearity property. However, the associated manifold is not stable; hence a random perturbation will result in the property 1 situation applying.

Property 4. If the initial positions of the agents are collinear but are otherwise generic, under the second law the agents will assume a common nonzero velocity exponentially fast.

\subsection{Control of a Minimally Persistent Formation with Leader and First Follower}

For this subsection, all graphs have at least $N \geq 3$ vertices. With the minimally persistent property, the edge count is precisely $2 N-3$. A further consequence of minimal persistence [9] is that vertices have an out-degree of at most 2, and apart from such vertices either there are exactly three vertices with an out-degree of 1 , or one vertex with an out-degree of zero and one with an out-degree of 1 . In the latter case, the zero out-degree vertex is termed a leader. The corresponding formation agent, having no neighbor from which its distance must be maintained, is not constrained at all in its motion. When the out-degree 1 vertex has the leader as its neighbor, it is termed a first follower. The graphs considered here are leader-first-follower graphs. It turns out that some results for minimally persistent graphs without a leader-firstfollower structure are, or appear to be, easily derivable from the result for a leader-first-follower structure. Hence leader-first-follower graphs are a suitable subclass to investigate initially. Also, in this subsection we restrict attention to graphs which contain one or more cycles, for reasons explained earlier.

Evidently before one could contemplate formation control for a nonminimally persistent graph, one would probably have to be able to treat minimally persistent graphs, and this might be the basis for treating nonminimally persistent graphs, though this has yet to be done.

By way of a final qualifying remark, in this subsection we are only able to present control laws for restoring a formation to its correct shape when it has undergone a small perturbation from its correct shape. The entire analysis is a linear one, and assumes that a linearized model is a valid approximation. Thus in comparison to the previous section, the vertex number is general but the convergence result is weaker.

We now explain the system model and outline the structure of the decentralized control law. We focus first on an agent $j$ which has two neighbors, agents $k$ and $m$, from which it must maintain its distance by amounts known to it. Suppose that the three agents concerned are all displaced from their nominal positions, call them $x_{0 j}, x_{0 k}, x_{0 m}$, by small amounts $\delta x_{j}, \delta x_{k}, \delta x_{m}$. 
Assuming it can measure the relative positions of its neighbor agents, agent $j$ determines to where it would need to move, in order to be at the correct distance from agents $k$ and $m$, (assuming the latter do not move while it is moving). Identify this target position as $x_{0 j}+\delta^{*} x_{j}$. Note that $\delta^{*} x_{j}$ is a function of $\delta x_{k}$ and $\delta x_{m}$. Agent $j$ actually moves to reduce the distance between where it currently is and this target position, by using the following law:

$$
\dot{\delta} \dot{x}_{j}=A_{j}\left(\delta^{*} x_{j}\left(\delta x_{k}, \delta x_{m}\right)-\delta x_{j}\right)
$$

with the following condition guaranteeing the distance reduction property

$$
A_{j}+A_{j}^{T}>0
$$

By simple geometric arguments, it is possible to express $\delta x_{j}^{*}$ in terms of $\delta x_{k}, \delta x_{m}$ and the nominal agent positions $x_{0 j}, x_{0 k}, x_{0 m}$. There results

$$
\delta \dot{x}_{j}=A_{j} B_{j}\left[\begin{array}{l}
\delta x_{j} \\
\delta x_{m} \\
\delta x_{k}
\end{array}\right]
$$

where $B_{j}$ is a matrix depending on differences of the position coordinates $x_{0 j}, x_{0 k}, x_{0 m}$, and is actually a submatrix of the rigidity matrix of the formation. Similar equations can be written for every agent, and for the leader and first follower. For details, the reader is referred to [13, 14]. Putting all equations together and with the first follower and leader labelled as vertices $N-1$ and $N$, there results:

$$
\frac{d}{d t}\left[\begin{array}{c}
\delta x_{1} \\
\delta x_{2} \\
\vdots \\
\delta x_{N}
\end{array}\right]=\Gamma\left[\begin{array}{c}
R \\
0_{3 \times 2 n}
\end{array}\right]\left[\begin{array}{c}
\delta x_{1} \\
\delta x_{2} \\
\vdots \\
\delta x_{N}
\end{array}\right]
$$

where $\Gamma$ is obtained by stacking together into a block diagonal matrix the 'gain' matrices $A_{j}$ each multiplied by a nonsingular $2 \times 2$ matrix, and $R$ is actually the rigidity matrix of the formation with agents at their nominal locations.

It is convenient to drop the last three rows of this equation (and the last three columns of $R$ ), to form a smaller size equation. The associated modes are attributable to the degrees of freedom $(D O F)^{4}$ of the leader and the first follower, which allow the whole formation to translate or rotate. They are not used for shape stabilization however. The key question now is: how should the entries of $\Gamma-$ a block diagonal matrix whose block entries are the $A_{j}$ multiplied by known $2 \times 2$ nonsingular matrices-be chosen, in order that the

\footnotetext{
${ }^{4}$ In $\Re^{2}$, a vertex has two, one or zero $\operatorname{DOF}(\mathrm{s})$ if it has no, one, or at least two outgoing edges; each outgoing edge uses up one DOF. A minor variation applies in 3 dimensions.
} 
linear equation set be stable. This will then ensure that when the agents of the formation are displaced a small distance from their nominal positions, the corrective action taken by each agent will drive the displacements to zero.

It is incidentally easy to find examples where the choice of $A_{j}=I$ is destabilizing. Thus the problem is nontrivial.

The key is the following result, $[13,22]$, which actually comes with a constructive proof:

Theorem 1 Consider the linear differential equation $\dot{x}=\Lambda A x$ in which a square real $A$ is prescribed and $\Lambda$ is diagonal, real and otherwise adjustable. Then a sufficient condition that there exists a choice of $\Lambda$ such that the equation is asymptotically stable, i.e. the eigenvalues of $\Lambda A$ all have negative real parts, is that the leading principal minors of $A$ are nonzero.

One can show further that full eigenvalue positionability is generically impossible via choice of $\Lambda$ in a number of cases where stabilization is possible.

The matrix $\Lambda$ of the theorem corresponds to $\Gamma$ in (8), minus its last three rows and columns. Notice that $\Gamma$, being block diagonal, has more adjustable entries than $\Lambda$, which is strictly diagonal, and it is an open question as to how to exploit this fact. The matrix $A$ of the theorem corresponds to the rigidity matrix $R$ less its last three columns. A far from trivial argument appealing to various properties of minimally persistent graphs, see [13, 22], guarantees the leading principal minors are nonzero (perhaps after vertex reordering).

Evidently, the preceding results should only be the first of many. We still need to know which formations are easy to control, and to understand how to exploit the freedom in the choice of control laws for each agent to achieve some kind of tradeoffs. We need results which are not just local or linearized, and we would like results which can deal with agents with their own dynamics. One can also envisage control laws other than memoryless ones. Actually, a particular decentralized proportional integral control scheme is presented in Section 5 for the particular applications case study of the chapter, cooperative surveillance with UAV formations.

\section{An Application: Surveillance with UAV Formations}

In this section, as an application domain of UAV formation control, we consider the task of cooperative surveillance over a 2-dimensional region of interest using a non-hierarchical formation of three autonomous UAVs. We derive a distributed control scheme for the formation to maintain a set of desired interagent distances within the formation while moving on a pre-defined path, under the assumption of constant UAV speeds. The particular formation control structure to be adopted is the asymmetric one described in Section 2.2, with the three-coleader form described in Section 3.1. However, note again that the individual control schemes of the agents in this section are designed in the proportional-integral form as opposed to the designs presented in Section 3.1. 


\subsection{System Specification}

The particular cooperative surveillance task to be considered is surveillance of a specified region of interest using a fleet of three UAVs with passive directionfinding sensor payloads, flying in an equilateral triangle formation.

This task is part of a research challenge problem posed by the Australian Defence Science and Technology Organisation (DSTO) with further specifications. One practical motivation for this research challenge is accurate cooperative localization of ground-based radar systems with small-size UAV fleets [23]. The cooperative surveillance task is planned to be experimented by DSTO using autonomous UAVs of the class Aerosonde, a small UAV developed by Aerosonde Pty Ltd [24]. An Aerosonde UAV typically has a wing span of $2.9 \mathrm{~m}$ and a maximum take-off mass of $15 \mathrm{~kg}$. It can stay flying for 8-30 hours, depending on the payload it carries, without refuelling.

The speed of each Aerosonde UAV, in the prospective formation flight test, is preset to a certain constant value (between $20 \mathrm{~m} / \mathrm{s}$ and $32 \mathrm{~m} / \mathrm{s}$ ) before take-off and is kept almost constant during the flight. However the constant speeds of different UAVs may be different, mainly due to different payloads. In our studies we assume a nominal constant speed of $32 \mathrm{~m} / \mathrm{s}$ and, in some cases, consider mild variations from this nominal value for some of the UAVs. Complying with the preset constant nominal speed of $32 \mathrm{~m} / \mathrm{s}$ and minimum turning radius of $400 \mathrm{~m}$, the maximum turning rate is specified to be $0.08 \mathrm{rad} / \mathrm{s}$.

The region of interest for surveillance is assumed to be a square with $30 \mathrm{~km}$ side length. For accuracy of localization of targets it is required to keep the inter-agent separation distances sufficiently large, and for coordination purposes the equilateral triangle formation shape and size is required to be maintained constant. Each of the three inter-agent distances is desired to be kept at $3 \mathrm{~km}$.

\subsection{Surveillance Task on a Spiral Path}

For the surveillance task described in Section 4.1, we consider a particular path to be followed by the center of mass (CM) of the formation, an Archimedean spiral path originating from the center of the $30 \mathrm{~km} \times 30 \mathrm{~km}$ region of interest. This spiral path can be formulated in time-indexed form in 2-dimensional polar coordinates $(r, \bar{\theta})$ corresponding to the cartesian coordinates $(x, y)=$ $(r \cos \bar{\theta}, r \sin \bar{\theta})$, with the center of the region of interest located at $(a, 0)$ (in both polar and cartesian coordinates), as

$$
\begin{aligned}
& r(t)=a+b \bar{\vartheta}(t) \\
& \bar{\theta}(t)=\bar{\vartheta}(t)(\bmod 2 \pi)
\end{aligned}
$$

where $\bar{\vartheta}(t)$ is a monotonically increasing function of $t$ satisfying $\bar{\vartheta}(0)=0$ and $\lim _{t \rightarrow \infty} \bar{\vartheta}(t)=\infty$, and the design constants $a, b \geq 0$ denote, respectively, the initial radial offset and the radial increase rate of the spiral. The main 
motivations for using a spiral path are as follows. The spiral path (9) originating from $(a, 0)$ (polar) scans the vicinity of $(a, 0)$ (polar) in a well-formulated polar form with constant increase rates of both the angle and radius. The periodicity rate of the scan angle $\bar{\theta}(t)$ (modulo $2 \pi$ ) and the increase rate of the scan radius $r(t)$ can be adjusted by selecting the spiral path parameters $a, b$ accordingly. An agent, actual or fictitious, following this path will have a continuously increasing turning radius and hence continuously decreasing turning rate.

Now, consider a fictitious or virtual point agent $A$ perfectly tracking the time-indexed spiral path (9) with constant speed $v(t)=v_{c}$ and agent kinematics

$$
\begin{aligned}
\dot{x}(t) & =v_{c} \cos (\theta(t)) \\
\dot{y}(t) & =v_{c} \sin (\theta(t)) \\
\dot{\theta}(t) & =\omega(t)
\end{aligned}
$$

where $p(t)=(x(t), y(t)), \theta(t)$, and $\omega(t)$ denote respectively the position, heading, and angular velocity of $A$ at time instant $t \geq 0$. In this chapter, we define the heading of an agent moving in 2-dimensions as the counter-clock-wise angle from the cartesian $x$-axis to the motion direction vector of the agent.

Matching the virtual agent kinematics (10) with the spiral path equation (9) in order to satisfy the perfect tracking requirement, we have

$$
\begin{aligned}
& x(t)=r(t) \cos (\bar{\theta}(t)) \\
& y(t)=r(t) \sin (\bar{\theta}(t)) \\
& \dot{x}(t)=-r(t) \dot{\bar{\vartheta}}(t) \sin (\bar{\theta}(t))+\dot{r}(t) \cos (\bar{\theta}(t)) \\
& \dot{y}(t)=r(t) \dot{\bar{\vartheta}}(t) \cos (\bar{\theta}(t))+\dot{r}(t) \sin (\bar{\theta}(t))
\end{aligned}
$$

Similarly, we have

$$
v(t)=v_{c}=|\dot{p}(t)|=\sqrt{\left.r^{2}(t) \dot{\bar{\vartheta}}^{2}(t)+\dot{r}(t)\right)^{2}}=\dot{\bar{\vartheta}}(t) \sqrt{(a+b \bar{\vartheta}(t))^{2}+b^{2}}
$$

noting that $\dot{r}(t)=b \dot{\bar{\vartheta}}(t)$. In order to satisfy (11), $\dot{\bar{\vartheta}}(t)$ needs to be chosen as

$$
\dot{\bar{\vartheta}}(t)=\frac{v_{c}}{\left((a+b \bar{\vartheta}(t))^{2}+b^{2}\right)^{1 / 2}}
$$

In the next section, we design a decentralized control scheme for the 3-UAV fleet mentioned in the beginning of this section to maintain the equilateral rigid formation described before while the CM of this formation is tracking the virtual agent $A$ moving according to (9)-(12).

\section{Non-Hierarchical Formation Control for Surveillance}

To design a decentralized control scheme meeting the spiral path tracking and formation control tasks specified in Section 4 we adopt the asymmetric control 
structure presented in Section 2.2. Furthermore, we assume the three-coleader formation structure presented in Section 3. Note here that a three-coleader (or non-hierarchical ${ }^{5}$ ) structure, as opposed to leader-follower (or hierarchical) structure, allows a balanced distribution of the tracking and formation maintenance tasks among the three agents (UAVs), and hence is expected to be more robust to speed variations of individual agents and atmospheric disturbances.

The formation maintenance and path tracking tasks are depicted in Fig. 1: The agents labelled as $A_{1}, A_{2}, A_{3}$, with respective positions $p_{1}(t), p_{2}(t), p_{3}(t)$ at each time instant $t$, are required to meet the inter-agent distance constraints $\left|p_{1}(t)-p_{2}(t)\right|=d_{12}=3 \mathrm{~km},\left|p_{2}(t)-p_{3}(t)\right|=d_{23}=3 \mathrm{~km}$ and $\left|p_{3}(t)-p_{1}(t)\right|=$ $d_{31}=3 \mathrm{~km}$, respectively, as well as keeping their distances to the virtual agent $A$ ( which is moving on the spiral path (9)) at $\left|p(t)-p_{i}(t)\right|=d_{i c}=\sqrt{3} \mathrm{~km}$ $(i \in\{1,2,3\})$ so that the formation CM traces (9).

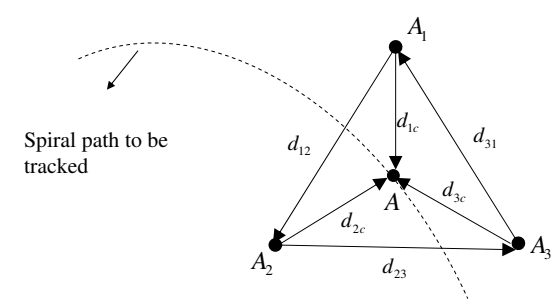

Fig. 1. Formation structure together with the formation control and path tracking tasks for the cooperative UAV surveillance task.

\subsection{Agent Models}

Each agent $A_{i}, i \in\{1,2,3\}$, in compliance with the specifications of Section 4 is assumed to move with a constant speed $v_{i}(t)=v_{c i}$ and agent kinematics

$$
\begin{aligned}
\dot{x}_{i}(t) & =v_{c i} \cos \left(\theta_{i}(t)\right) \\
\dot{y}_{i}(t) & =v_{c i} \sin \left(\theta_{i}(t)\right) \\
\dot{\theta}_{i}(t) & =\omega_{i}(t)
\end{aligned}
$$

where $p_{i}(t)=\left(x_{i}(t), y_{i}(t)\right)$, and $\theta_{i}(t)$ and $\omega_{i}(t)$ are respectively the heading and angular velocity of $A_{i}$ at time instant $t \geq 0$. In the sequel we consider

\footnotetext{
${ }^{5}$ Here, the term "hierarchy" is used in terms of leading/following (or path tracking/distance keeping) task distribution. For minimally persistent formations with three agents and 3-coleader structure, as opposed to the leader-follower case, note that this task distribution is uniform among the agents and hence there is no hierarchy in the above sense.
} 
both of the cases (i) where $v_{c 1}=v_{c 2}=v_{c 3}$ and (ii) where $v_{c i}$ are different, and we demonstrate for case (ii) that there exist sensible upper bounds $\bar{\epsilon}_{1}, \bar{\epsilon}_{2}>0$ for which the formation maintenance is feasible, despite the speed difference between agents, if $\left|v_{c 1}-v_{c 2}\right|<\bar{\epsilon}_{1}$ and $\left|v_{c 1}-v_{c 3}\right|<\bar{\epsilon}_{2}$.

Each $A_{i}$ is assumed to sense the location of itself as well as the agent it follows. Furthermore each $A_{i}$ is assumed to know the spiral path to be tracked by the formation $\mathrm{CM}$ and hence the time trajectory $p(t)$ of the virtual agent A.

\subsection{Control Design}

We consider a non-hierarchical decentralized control design, i.e. we design three identical individual agent controllers, one for each of the agents $A_{1}, A_{2}, A_{3}$. For agent $A_{i} \quad(i \in\{1,2,3\})$, the individual controller inputs at each time instant $t \geq 0$ are the locations $p_{i}(t), p_{j}(t), p(t)$ of, respectively, itself, the agent $A_{j}$ it follows, and the virtual agent $A$ introduced in Section 4.2. The controller output is the angular velocity $\omega_{i}(t)$ in (13). The corresponding feedback control law is designed in the proportional-integral (PI) form with proportional gain $k_{P}>0$ and integrator gain $k_{I}>0$ as

$$
\omega_{i}(t)=\dot{\theta}_{i}(t)=k_{P}\left[\theta_{i d}(t)-\theta_{i}(t)\right]+k_{I} \int_{t_{0}}^{t}\left[\theta_{i d}(t)-\theta_{i}(t)\right] d t
$$

where generation of the desired heading signal $\theta_{i d}(t)$ is explained in the sequel in detail. In short, $\theta_{i d}(t)$ corresponds to the agent motion direction that would bring $A_{i}$ to a location $p_{i d}(t)$ with distance $d_{i j}$ to $p_{j}(t)$ and distance $d_{i c}$ to $p(t)$.

The desired location $p_{i d}(t)$ is calculated, similarly to $[25,26]$, using the following circle intersection rule:

$$
p_{i d}(t)=\arg \min \left\{\left\|p-p_{i}(t)\right\| \mid p \in C\left(p(t), d_{i c}\right) \cap C\left(p_{j}(t), d_{i j}\right)\right\}
$$

where the notion $C(\cdot, \cdot)$ is used to formulate circles with the first argument denoting the center and the second denoting the radius, and it is assumed that $C\left(p(t), d_{i c}\right) \cap C\left(p_{j}(t), d_{i j}\right)$ is non-empty. The case of an empty intersection is dealt with later.

The corresponding desired heading signal $\theta_{i d}(t)$ is generated using

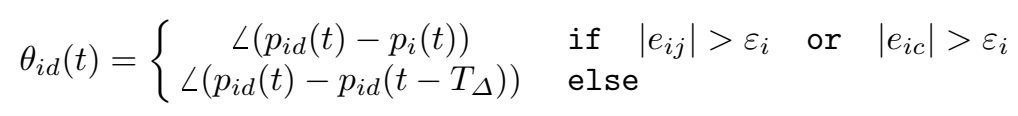

where $T_{\Delta}>0$ is a certain delay term used for interpolation,

$$
\begin{aligned}
& e_{i j}=\left\|p_{i}(t)-p_{j}(t)\right\|-d_{i j}, \\
& e_{i c}=\left\|p_{i}(t)-p_{c}(t)\right\|-d_{i c},
\end{aligned}
$$

and $\varepsilon_{i}$ is a separation tolerance term. In our design and simulation studies, the values of the delay and tolerance terms are taken as $T_{\Delta}=1 \mathrm{sec}$. and $\varepsilon_{i}=30 \mathrm{~m}$. 
The switching law (16) determines the desired direction of motion to be from the current position of agent $A_{i}$ towards the closest intersection point of the two circles $C\left(p(t), d_{i c}\right)$ and $C\left(p_{j}(t), d_{i j}\right)$ if either of the two separation errors $\left|e_{i j}\right|,\left|e_{i c}\right|$ is larger than the tolerated value $\varepsilon_{i}$, and in a direction parallel to that in which $p_{i d}$ is estimated to be moving otherwise.

\section{Simulation-Based Analysis of the Control Laws}

The decentralized control scheme developed in Section 5 is numerically analyzed using a set of MATLAB/Simulink simulations. For all conducted simulations the parameters of the spiral path (9) are taken as $a=0$ and $b=\frac{6000}{2 \pi}$ and, as mentioned in Section 5, the desired separation distances are set to $d_{12}=d_{23}=d_{31}=3 \mathrm{~km}$ and $d_{i c}=\sqrt{3} \mathrm{~km}(i \in\{1,2,3\})$. The area to be surveyed is assumed to lie in a $30 \mathrm{~km} \times 30 \mathrm{~km}$ square region.

Three cases are considered: (i) UAVs are all flying at the same constant speed and there exists no actuator or sensor noise affecting the system. (ii) UAVs are flying at different constant speeds and there exists no actuator or sensor noise affecting the system. (iii) UAVs are all flying at the same constant speed and there exist some actuator and sensor noises affecting the system. We present the simulation results and discussions for these three cases separately in the following subsections.

\subsection{Ideal Motion Behavior}

In this case we assume that all the UAVs are flying at the same constant speed of $32 \mathrm{~m} / \mathrm{s}$ and there exists no actuator or sensor noise affecting the system. The decentralized control laws (14)-(16) are applied using the design parameters $k_{P}=2, k_{I}=0.0005, \varepsilon_{i}=30 \mathrm{~m}$. The simulation results shown in Fig. 2 demonstrate that both the path tracking and formation maintenance tasks are successfully achieved.

\subsection{Effects of Speed Variations}

In this simulation case we assume that UAVs are flying at different constant speeds around a nominal value of $32 \mathrm{~m} / \mathrm{s}$ and there exists no actuator or sensor noise affecting the system.

In the control design presented in Section 5 , it is assumed that the two circles $C\left(p(t), d_{i c}\right)$ and $C\left(p_{j}(t), d_{i j}\right)$ always intersect, which is a valid assumption for the ideal case presented in Section 6.1. However, it is observed in simulation studies that this assumption may be violated occasionally if the differences between UAV speeds are sufficiently large. To circumvent such occasional cases, the control law (16) is modified as follows: 

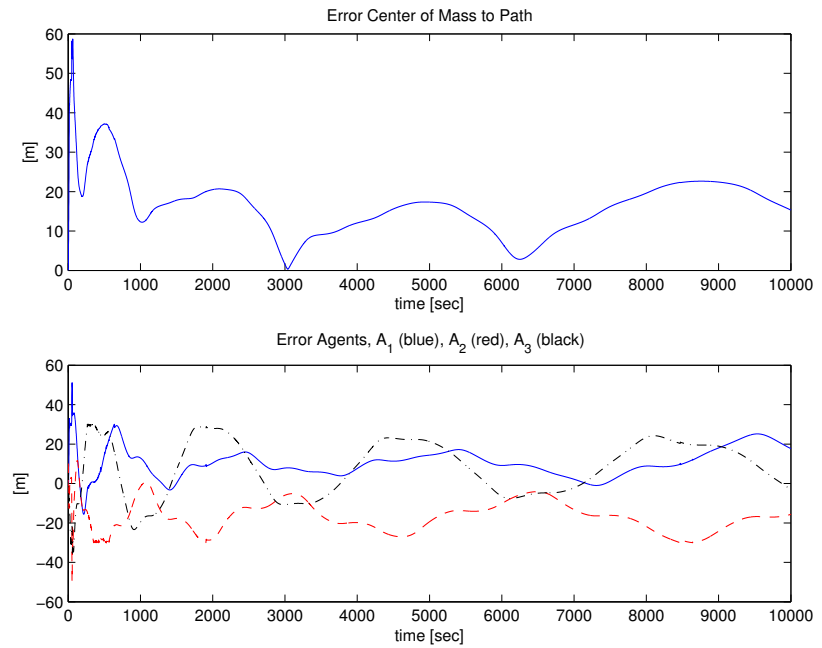

Fig. 2. Inter-agent distance keeping errors and the distance of CM to the path to be tracked.

$$
\theta_{i d}(t)=\left\{\begin{array}{cl}
\angle\left(p_{i}(t)-p(t)\right) & \text { if }\left\|p(t)-p_{i}(t)\right\|<d_{i j}-d_{i c} \\
\angle\left(p(t)-p_{i}(t)\right) & \text { else if }\left\|p(t)-p_{i}(t)\right\|>d_{i j}+d_{i c} \\
\angle\left(p_{i d}(t)-p_{i}(t)\right) & \text { else if }\left|e_{i j}\right|>\varepsilon_{i} \text { or }\left|e_{i c}\right|>\varepsilon_{i} \\
\angle\left(p_{i d}(t)-p_{i d}\left(t-T_{\Delta}\right)\right) & \text { else }
\end{array}\right.
$$

The modified law (17) is the same as (16) when the circles $C\left(p(t), d_{i c}\right)$ and $C\left(p_{j}(t), d_{i j}\right)$ intersect. When they do not intersect, (17) defines the desired heading for agent $A_{i}$ to be towards $p(t)$ if it is too distant to the virtual agent $A$, and away from $p(t)$ if it is too close to $A$.

The modified control scheme (14)-(17) is applied to various simulation settings with different UAV speeds, where $A_{1}$ is always assigned a constant speed of $32 \mathrm{~m} / \mathrm{sec}$ and each of $A_{2}$ and $A_{3}$ is assigned a constant speed between $29 \mathrm{~m} / \mathrm{s}$ and $32 \mathrm{~m} / \mathrm{s}$. Fig. 3 shows the root-mean-square of the error

$$
\sqrt{\frac{1}{t_{f}} \int_{0}^{t_{f}}\left(\frac{p_{1}(t)+p_{2}(t)+p_{3}(t)}{3}-p(t)\right)^{2} d t}
$$

versus $v_{c 2}$ and $v_{c 3}$, where $t_{f}$ is the final time of surveillance. This figure demonstrates that agent speed differences within a certain limit are allowable in meeting the path tracking and formation maintenance tasks.

\subsection{Effects of Sensor and Actuator Disturbances}

UAVs same speed, sensor and actuator disturbance 


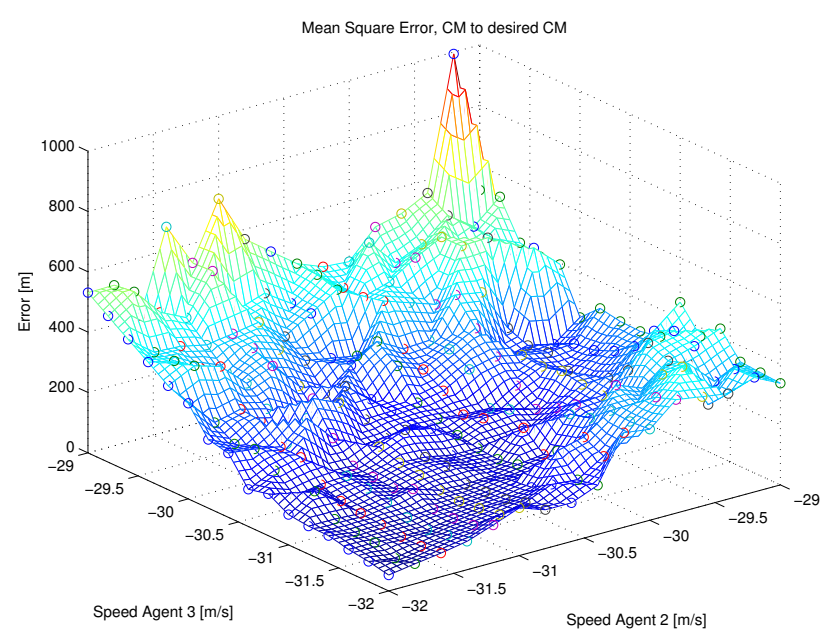

Fig. 3. Root-mean-square of the distance between the actual and desired locations of the formation CM versus agent speeds $v_{c 2}$ and $v_{c 3}$.

In this final case, we assume that the three UAVs are all flying at the same constant speed and there exist some actuator and sensor noises affecting the system. The cumulative actuator and sensor noises are represented, respectively, by the parameters $\gamma_{a}$ and $\gamma_{s}$ represent in Fig. 4 depicting the diagram of the individual controllers of the agents. Various simulations have been per-

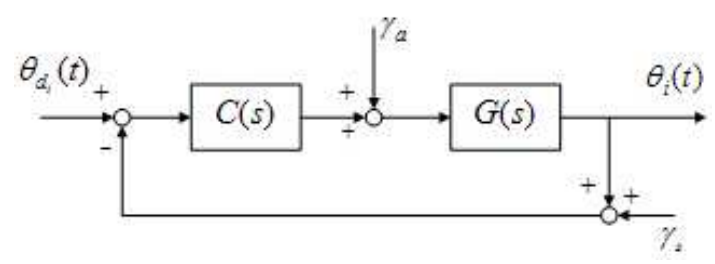

Fig. 4. Block diagram of the individual controller of agent $A_{i}$ in the existence of actuator and sensor noises.

formed with different standard deviation values for $\gamma_{a}$ and $\gamma_{s}$, modelling the value of each of these two noises at any time instant as zero-mean Gaussian random variable. The results of these simulations are shown in Fig. 5, which demonstrates that actuator and sensor noises within a certain limit are allowable in meeting the path tracking and formation maintenance tasks. 


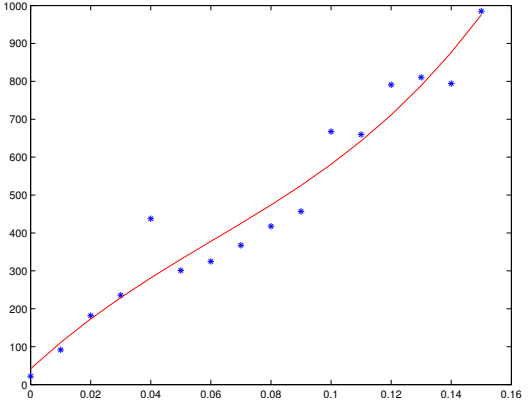

(a)

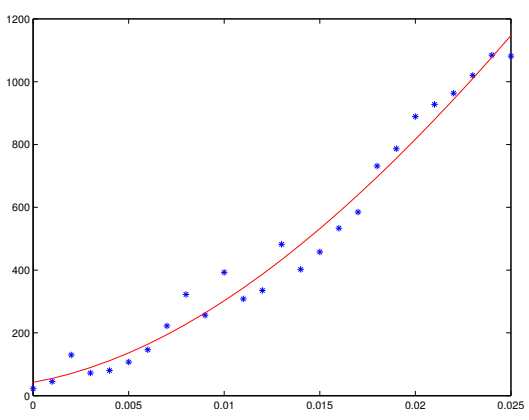

(b)

Fig. 5. Root-mean-square of the distance between the actual and desired locations of the formation CM versus standard deviations of (a) the actuator noise $\gamma_{a}$ and (b) the sensor noise $\gamma_{s}$.

\section{Concluding Remarks}

In the first part of this chapter we have described how to characterize the choice of agent pairs to secure this shape-preserving property for a planar formation based mainly on graph theory and linear algebra. We have described decentralized control laws which will stably restore the shape of a formation when the distances between nominated agent pairs become unequal to their prescribed values.

Some possible future research directions based on the first part of the chapter are as follows: In the ideal world of point agents, be it in two dimensions or three dimensions, it would be helpful to identify the class of decentralized control laws that could maintain formation shape. Of course, one must agree first on what variables are to be controlled, and here we have emphasized distance, and in the case of controlling distances, one must agree on what agent has the responsibility (or possibly shares the responsibility) for controlling what distance. Distance is naturally not the only variable, and other variables should be contemplated. It would be very helpful also to understand what formations were easy to control and what formations were hard to control: one that was easy to control would presumably require just small control signals to restore the formation shape when perturbed away from its ideal, and would be comparatively insensitive to noise perturbing the measurements. These questions also need to be posed when significant agent dynamics are introduced into the model; when there are speed, turning rate and sensing radius constraints (as in the second part of the chapter); and when there are constraints on sensing involving typically blind cones (an agent may not be allowed to have "eyes in the back of its head").

In the second part of the chapter, we have considered a particular application problem of flying a group of three UAVs in an equilateral triangle formation, with the center of mass following a nominated trajectory reflect- 
ing constraints on turning radius, and with a requirement that the speeds of the UAVs are constant. We have proposed a decentralized non-hierarchical proportional-integral control scheme for this particular problem. The proposed control scheme is tested in various simulation scenarios to examine the basic characteristics of the proposed control scheme for the case where the UAV speeds are all equal as well as the affects of sensor and actuator disturbances and differences between the UAV speeds on the system performance. There is an ongoing study on analyzing the affects of the wind in details, even though this is partially covered within the context of sensor and actuator disturbances. The simulation results demonstrate the success and effectiveness of the proposed scheme within certain bounds of disturbances and UAV speed differences.

A particular future research topic related to the second part of the chapter is comprehensive mathematical analysis of the stability and robustness properties of the proposed scheme and mathematical explanation of the observed behavior of the path tracking error of the formation center of mass and the inter-agent distance keeping errors. A more practical research direction is revision of the control laws for further robustification against sensor and actuator disturbances and UAV speed differences and enhancement of the path tracking and formation maintenance performance.

\section{References}

1. S. Hubbard, B. Babak, S. Sigurdsson, and K. Magnusson, "A model of the formation of fish schools and migrations of fish," Ecological Modelling, vol. 174, pp. 359-374, 2004.

2. S. Janson, M. Middendorf, and M. Beekman, "Honey bee swarms: How do scouts guide a swarm of uninformed bees?," Animal Behaviour, vol. 70, no. 1, pp. 349$358,2005$.

3. J. Shao, G. Xie, and L. Wang, "Leader-following formation control of multiple mobile vehicles," IET Control Theory and Applications, vol. 1, pp. 545-552, March 2007.

4. T. Tay and W. Whiteley, "Generating isostatic frameworks," Structural Topology, no. 11, pp. 21-69, 1985.

5. B. Jackson and T. Jordan, "Connected rigidity matroids and unique realizations of graphs," Journal of Combinatorial Theory, vol. B, no. 94, pp. 1-29, 2004.

6. R. Olfati-Saber and R. M. Murray, "Distributed cooperative control of multiple vehicle formations using structural potential functions," in Proc. of the 15th IFAC World Congress, (Barcelona, Spain), pp. 1-7, 2002.

7. T. Eren, W. Whiteley, A. S. Morse, P. N. Belhumeur, and B. D. Anderson, "Sensor and network topologies of formations with direction, bearing and angle information between agents," in Proceedings of the 42nd IEEE Conference on Decision and Control, (Maui, Hawaii), pp. 3064-3069, December 2003.

8. Z. Lin, B. Francis, and M. Maggiore, "Necessary and sufficient graphical conditions for formation control of unicycles," IEEE Trans. on Automatic Control, vol. 50, pp. 121-127, January 2005. 
9. C. Yu, J. Hendrickx, B. Fidan, B. Anderson, and V. Blondel, "Three and higher dimensional autonomous formations: Rigidity, persistence and structural persistence," Automatica, pp. 387-402, March 2007.

10. G. Laman, "On graphs and rigidity of plane skeletal structures," J. Engrg. Math., vol. 4, pp. 331-340, 1970.

11. B. Anderson, C. Yu, B. Fidan, and J. Hendrickx, "Control and information architectures for formations," in Proc. IEEE International Conference on Control Applications, vol. 56, (Munich, Dermany), pp. 1127-1138, October 2006.

12. J. Hendrickx, B. Anderson, J.-C. Delvenne, and V. Blondel, "Directed graphs for the analysis of rigidity and persistence in autonomous agent systems," International Journal of Robust Nonlinear Control, vol. 17, pp. 960-981, July 2007.

13. C. Yu, B. Anderson, S. Dasgupta, and B. Fidan, "Control of minimally persistent formations in the plane," Submitted for publication, December 2006.

14. B. Anderson, C. Yu, S. Dasgupta, and A. Morse, "Control of a three coleaders formation in the plane," Systems $\&$ Control Letters, vol. 56, pp. 573-578, 2007.

15. M. Cao, A. Morse, C.Yu, B. Anderson, and S. Dasgupta, "Controlling a triangular formation of mobile autonomous agents," To appear in IEEE Conference on Decision and Contol, 2007.

16. R. Olfati-Saber and R. M. Murray, "Distributed cooperative control of multiple vehicle formations using structural potential functions," in Proc. of the 15th IFAC World Congress, (Barcelona, Spain), pp. 1-7, 2002.

17. R. Olfati-Saber and R. M. Murray, "Graph rigidity and distributed formation stabilization of multi- vehicle systems," in Proc of the 41st IEEE Conf. on Decision and Control, (Las Vegas, NV), pp. 2965-2971, 2002.

18. L. Krick, "Application of graph rigidity in formation control of multi-robot networks," Master's thesis, Department of Electrical and Computer Engineering, University of Toronto, 2007.

19. D. Paley, N. E. Leonard, and R. Sepulchre, "Collective motion: bistability and trajectory tracking," in Proc. of the 43rd IEEE Conference on Decision and Control, vol. 2, pp. 1932-1937, 2004.

20. E. W. Justh and P. S. Krishnaprasad, "Equilibria and steering laws for planar formations," Systems and Control Letters, vol. 52, no. 1, pp. 25-38, 2004.

21. S. L. Smith, M. E. Broucke, and B. A. Francis, "Stabilizing a multi-agent system to an equilibrium polygon formation," in Proc. 17th International Symposium on Mathematical Theory of Networks and Systems, pp. 2415-2424, 2006.

22. B. Anderson, S. Dasgupta, and C. Yu, "Control of directed formations with leader-first follower structure," To appear in IEEE Conference on Decision and Contol, 2007.

23. S. Drake, K. Brown, J. Fazackerley, and A. Finn, "Autonomous control of multiple uavs for the passive location of radars," Tech. report, Defence Science and Technology Organisation, pp. 403-409, 2005.

24. D. Ledger, "Electronic warfare capabilities of mini UAVs," in Proc. the Electronic Warfare Conference, (Kuala Lumpur), 2002.

25. S. Sandeep, B. Fidan, and C. Yu, "Decentralized cohesive motion control of multi-agent formations," Proc. 14th Mediterranean Conference on Control and Automation, June 2006.

26. B. Fidan, B. Anderson, C. Yu, and J. Hendrickx, Modeling and Control of Complex Systems, ch. Persistent Autonomous Formations and Cohesive Motion Control, pp. 247-275. Taylor \& Francis, 2007. 